\title{
Magnetic resonance imaging-guided attenuation and scatter corrections in three-dimensional brain positron emission tomography
}

\author{
Habib Zaidi, ${ }^{\text {a) }}$ Marie-Louise Montandon, and Daniel O. Slosman \\ Division of Nuclear Medicine, Geneva University Hospital, CH-1211 Geneva 4, Switzerland
}

(Received 29 October 2002; accepted for publication 3 March 2003; published 22 April 2003)

\begin{abstract}
Reliable attenuation correction represents an essential component of the long chain of modules required for the reconstruction of artifact-free, quantitative brain positron emission tomography (PET) images. In this work we demonstrate the proof of principle of segmented magnetic resonance imaging (MRI)-guided attenuation and scatter corrections in three-dimensional (3D) brain PET. We have developed a method for attenuation correction based on registered T1-weighted MRI, eliminating the need of an additional transmission (TX) scan. The MR images were realigned to preliminary reconstructions of PET data using an automatic algorithm and then segmented by means of a fuzzy clustering technique which identifies tissues of significantly different density and composition. The voxels belonging to different regions were classified into air, skull, brain tissue and nasal sinuses. These voxels were then assigned theoretical tissue-dependent attenuation coefficients as reported in the ICRU 44 report followed by Gaussian smoothing and addition of a good statistics bed image. The MRI-derived attenuation map was then forward projected to generate attenuation correction factors (ACFs) to be used for correcting the emission (EM) data. The method was evaluated and validated on 10 patient data where TX and MRI brain images were available. Qualitative and quantitative assessment of differences between TX-guided and segmented MRIguided 3D reconstructions were performed by visual assessment and by estimating parameters of clinical interest. The results indicated a small but noticeable improvement in image quality as a consequence of the reduction of noise propagation from TX into EM data. Considering the difficulties associated with preinjection TX-based attenuation correction and the limitations of current calculated attenuation correction, MRI-based attenuation correction in 3D brain PET would likely be the method of choice for the foreseeable future as a second best approach in a busy nuclear medicine center and could be applied to other functional brain imaging modalities such as SPECT. (C) 2003 American Association of Physicists in Medicine. [DOI: 10.1118/1.1569270]
\end{abstract}

Key words: PET, attenuation correction, scatter correction, MRI, brain imaging

\section{INTRODUCTION}

To improve the capability of positron emission tomography (PET) for investigating the living human brain with regard to blood flow, metabolism and receptor characteristics for small structures, both the spatial resolution and quantitative accuracy have to be improved compared to what the commercial scanners' hardware and software can provide at the present time. To reach these research goals, many attempts are being made by different scanner manufacturers and research groups to design new next generation high-resolution 3D PET tomographs with depth of interaction information capabilities. ${ }^{1,2}$ On the other hand, despite the worthwhile research performed in the area of quantification, the development of improved models for accurate attenuation, scatter and partial volume effect corrections dedicated to functional brain imaging is still an open question which requires further research and development efforts. ${ }^{3}$

Reliable attenuation correction methods for quantitative $3 \mathrm{D}$ PET require accurate delineation of both the head contour and spatial distribution of attenuation coefficients. To this end, different approaches to determine the attenuation map have been described in the literature. ${ }^{4}$ Basically, two broad classes of methods have emerged: calculated (trans- missionless) approaches and transmission-based approaches. The later represents actually the gold standard particularly in research studies where there is greater emphasis on accurate quantitative procedures. Among the first class of methods, the manual fit-ellipse method has found success in clinical routine for simplicity reasons. Automated contour detection methods allow for a certain thickness of higher attenuation material to be added to the calculation to account for the skull. ${ }^{5-8}$ A more sophisticated method computes a threecomponent attenuation map for brain PET imaging. ${ }^{9}$ The technique generates an estimated skull image by filtered backprojection of the reciprocal of an emission sinogram. The thickness and radius of the skull is estimated from profiles extracted from the image. The resulting thickness and radius values are then used to generate a model of the brain, skull, and scalp. Appropriately scaled linear attenuation coefficients are then assigned to estimate the attenuation map for the head. Stodilka et al. ${ }^{10}$ developed a method of inferring-attenuation distributions by deformably registering the brain component of a digital head atlas ${ }^{11}$ to SPECT images. More recently, a new approach to derive 3D patientspecific head contour dedicated to functional brain imaging using an optical tracking system has been described. ${ }^{12}$ Based 
on this contour, a previously acquired reference attenuation map is transformed to match the contour of the reference head with the target head using the thin plate spline technique. A practical advantage of the optical tracking system is that it can also be utilized for motion correction.

While preinjection transmission scanning in PET is used clinically in many centers, it is of questionable value for patients with neurodegenerative disease due to misregistration errors between emission (EM) and transmission (TX) scanning resulting from patient mispositioning and problems associated with patient motion. The advent of multimodality imaging systems has revolutionized the practice of nuclear medicine. One of the major advantages of combined PET/CT systems is that PET data are intrinsically aligned to anatomical information from the x-ray CT without the use of external markers or internal landmarks. Quantification is also improved by using the low noise CT transmission information during the correction of the PET data for self-attenuation and for contamination from scattered photons. ${ }^{13}$ On the other hand, combining PET with magnetic resonance imaging (MRI) technology is scientifically more challenging owing to the strong magnetic fields. Nevertheless, significant progress has been made resulting in the design of a prototype small animal PET scanner with lutetium oxyorthosilicate (LSO) detector blocks of $3.8 \mathrm{~cm}$ ring diameter coupled to three multichannel photomultipliers via optical fibers ${ }^{14}$ so that the PET detector can be operated within a conventional MR system. The authors reported no appreciable artifacts caused by the scintillators in the MR images. A second larger $(11.2 \mathrm{~cm})$ prototype is being developed for simultaneous PET/MR imaging of mice and rats at different magnetic field strengths. ${ }^{15}$ While the advantages of combined PET/CT could in principle be replicated by combined PET/MRI, the usefulness of MRI for attenuation correction in radionuclide imaging is not yet established. ${ }^{16}$ This paper aims to investigate the feasibility of using segmented MRI for attenuation correction in brain PET. Since MR images are generally available for patients undergoing cerebral PET scanning, we have developed a method using segmented MR images to generate a patientspecific attenuation map. ${ }^{17}$ The validity of the approach was tested on 10 patients admitted for evaluation of surgical relief of their epilepsy.

\section{MATERIALS AND METHODS}

\section{A. Algorithm description}

The algorithm used for attenuation and scatter corrections combines data obtained from MRI and PET to generate a patient-specific attenuation map. Figure 1 shows a flow chart of the general principles of the method. The basic steps followed when applying the method consist of the following.

(1) Acquire brain PET and T1-weighted MRI data for each patient.

(2) Preliminary 3D PET reconstruction after scatter and uniform fit-ellipse based calculated attenuation corrections.

(3) Extraction of brain tissues from MR images by removing skull and scalp (extracranial) tissues.

(4) Registration of MRI to PET images.
(5) Record spatial transformation matrix for brain MRI.

(6) Apply same spatial transformation to original MR images.

(7) Segment the MR images to five regions: air, brain tissue, skull, nasal sinuses, and scalp.

(8) Merge the brain and scalp tissues (since they have the same attenuation properties).

(9) Assignment of attenuation coefficients to the four regions followed by Gaussian smoothing that approximates the resolution of the PET scanner $(6 \mathrm{~mm}$ full width at half-maximum).

(10) Addition of a good statistics bed image after adjustments to account for different bed elevations.

(11) Segmented MRI attenuation map-guided scatter correction using the single-scatter simulation (SSS) technique.

(12) Forward project the attenuation map to generate ACFs by taking the inverse natural logarithm.

(13) Nonuniform attenuation correction using the segmented MRI-based attenuation map.

(14) 3D brain PET reconstruction using the 3DRP algorithm.

The total data processing time for the whole procedure required to generate the MRI-guided attenuation map was less than 10 min on a Sun SPARC station 60, which is comparable to the time required to delineate manually the elliptical ROIs when using calculated attenuation correction. The whole process is illustrated in Fig. 2. The extracranial tissues were removed from MR images prior to applying the coregistration algorithm using an automated extraction technique. The preliminary 3D PET reconstruction includes modelbased scatter correction and calculated attenuation compensation since this has been shown to improve registration accuracy.$^{10}$ MRI-PET image registration was carried out using the Automated Image Registration (AIR 3.03) software package, a general linear intermodality and intramodality (within or across subjects, 2D or 3D) image registration tool. ${ }^{18}$ After applying the recorded spatial transformation, the original MR images are segmented using a Fuzzy C-Means (FCM) algorithm. ${ }^{19}$ This fuzzy clustering segmentation algorithm is unsupervised, adaptive and allows the classification of brain tissues into main components in terms of soft tissue contrast. FCM has been successfully used in the soft or fuzzy segmentation of MR images, as well as for the estimation of partial volumes. ${ }^{20}$ The algorithm clusters the image by computing a measure of membership (fuzzy membership) at each voxel for a specified number of classes. The fuzzy membership function, constrained to be between zero and one, reflects the degree of similarity between the data value at the location and the prototypical data value or centroid of its class. Thus, a high membership value near unity signifies that the data value at that location is close to the centroid for that particular class. Every voxel is then assigned to the cluster center for which it has the maximum membership degree. The algorithm has been largely tested on different data sets, acquired under various protocols. ${ }^{17,21}$ Our experiments were valuable to optimize the initial number of clusters. This overestimation of the actual regions present in the image greatly 


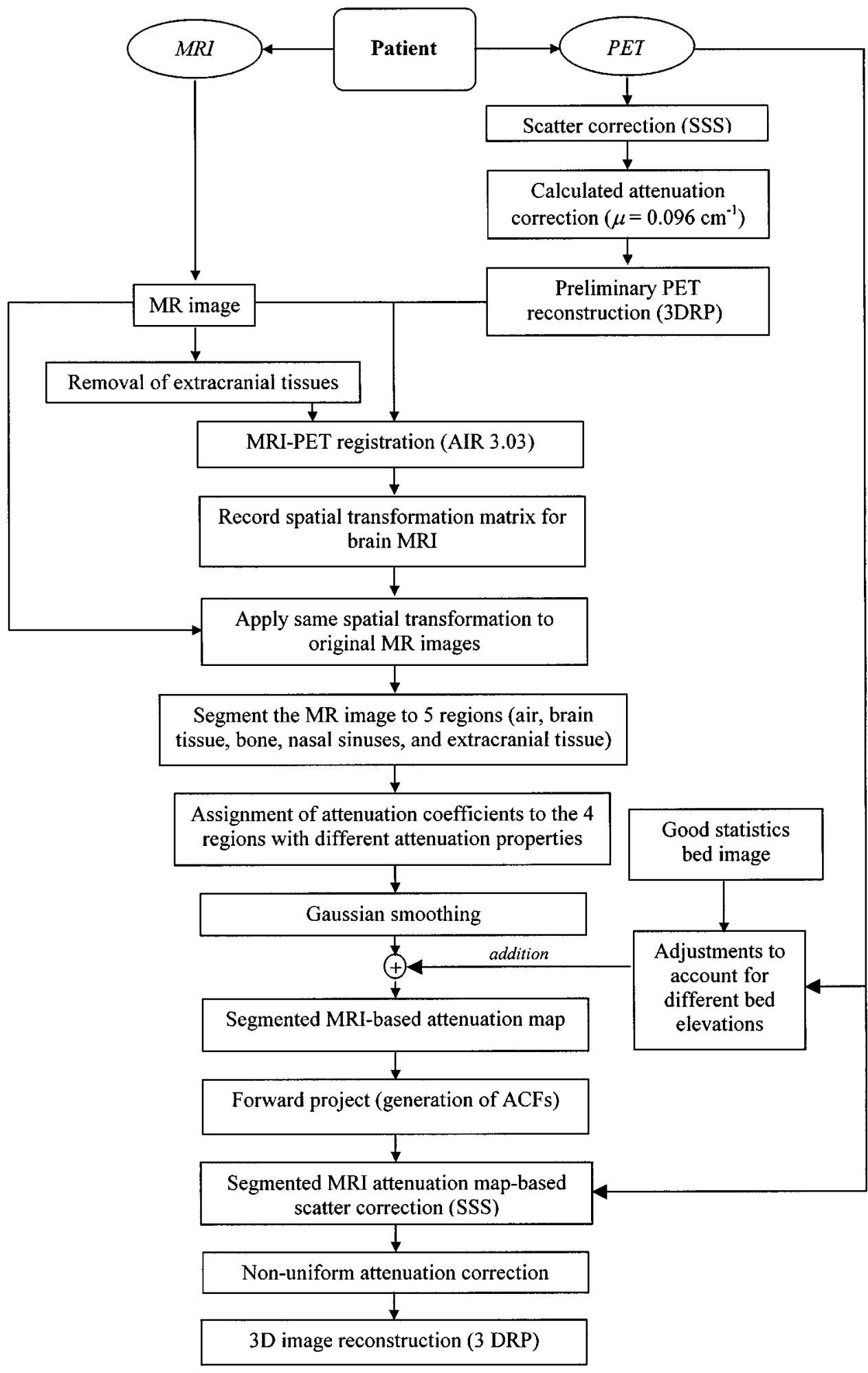

FIG. 1. Flow-chart of the algorithm summarizing the different steps needed to generate an attenuation map from segmented MR images and application of the attenuation and scatter correction procedures to PET data. Scatter correction is performed using a fast implementation of the singlescatter simulation (SSS) technique and 3D reconstruction carried out using the reprojection (3DRP) algorithm. simplifies the merging process ending up with four clusters. A contour detection algorithm is used to identify the external boundary of the head. This gives back a binary image, where the brain tissues have one label and the background and nasal sinuses share another one. In the final step, the nasal sinuses are identified using a labeling technique as outlined in Boudraa et al., ${ }^{22}$ taking advantage of the fact that voxels belonging to the nasal sinuses are totally surrounded by the head contour. A fast and efficient algorithm for image volume scanning and edge filling that avoids testing the condition on each voxel is used. ${ }^{21}$ The scalp and brain tissues are considered as a single class since they have similar attenuation properties. ${ }^{9}$ It is worth emphasizing that the difficulties associated with automatic segmentation of the skull on the T1-weighted spin-echo images using the FCM algorithm led to some manual intervention of the operator. This consists in 


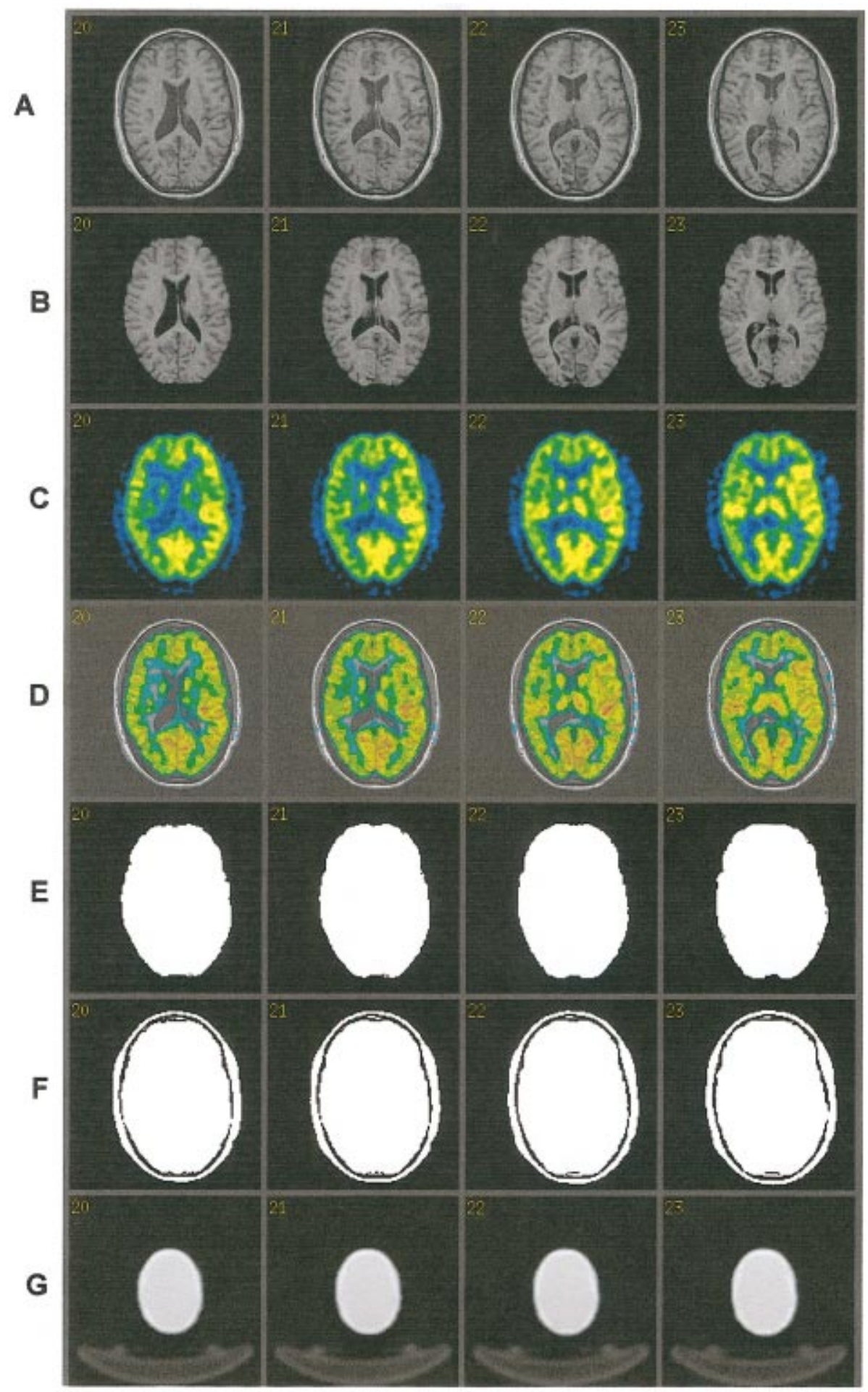

FIG. 2. Illustration of MRI-PET image registration and fusion using the AIR algorithm as well as brain T1-weighted MR image segmentation process and construction of the MRI-guided attenuation map. (A) Original MR images. (B) Brain tissues extracted from the MR images shown in (A) obtained by removing the skull and other extracranial tissues. (C) The preliminary PET images reconstructed with calculated uniform attenuation and scatter corrections (fit-ellipse contour delineation of the head with constant $\mu=0.096 \mathrm{~cm}^{-1}$ ). (D) Result of fusion between MRI and PET image data after coregistration and application of the spatial transformation to original MR images. (E) Segmented brain followed by edge filling giving back a binary image, where brain tissues have one label and the background and nasal sinuses share another one. (F) Addition of the segmented skull and scalp to (E). (G) Final segmented MRI-guided attenuation map after applying a Gaussian filter and adding up the bed image.

filling the complexly shaped skull base using a morphological "closing" operation to make it more uniform.

A good statistics transmission bed scan was acquired and the images reconstructed using a two-dimensional filtered backprojection algorithm similar to the protocol used for patient transmission scans reconstruction in clinical routine. This low noise image of the bed is added up to the final segmented MRI-guided attenuation map. The program reads 
TABLE I. Summary of linear attenuation coefficients (in $\mathrm{cm}^{-1}$ ) reported in the literature for different tissues of the head used for attenuation correction purposes in both SPECT $(140 \mathrm{keV})$ and PET $(511 \mathrm{keV})$. The assumptions made regarding the skull thickness are also shown.

\begin{tabular}{|c|c|c|c|c|c|}
\hline & \multicolumn{3}{|c|}{ Linear attenuation coefficient $\left(\mathrm{cm}^{-1}\right)$} & \multirow[b]{2}{*}{ Skull thickness (mm) } & \multirow[b]{2}{*}{ References } \\
\hline & Brain tissue & Skull & Nasal sinuses & & \\
\hline \multirow[t]{2}{*}{ SPECT $(140 \mathrm{keV})$} & 0.15 & 0.25 & 0.075 & $\mathrm{ps}^{\mathrm{a}}$ & 10 \\
\hline & 0.153 & 0.251 & 0.0942 & $\mathrm{na}^{\mathrm{b}}$ & 25 \\
\hline \multirow[t]{6}{*}{ PET (511 keV) } & 0.096 & 0.151 & na & 4.5 & 5 \\
\hline & 0.094 & 0.171 & na & 8 & 6 \\
\hline & 0.088 & 0.157 & na & $6 \%$ of the outer contour & 7 \\
\hline & 0.095 & 0.151 & na & 5 & 8 \\
\hline & 0.099 & 0.136 & na & ps & 9 \\
\hline & 0.0993 & 0.143 & 0.0536 & ps & This work \\
\hline
\end{tabular}

${ }^{a}$ Patient specific.

${ }^{\mathrm{b}}$ Not applicable.

from the header files of both images the vertical position of the bed and makes appropriate adjustments to account for the different bed elevations between the bed scan and the clinical brain scan.

\section{B. Assignment of attenuation coefficients}

The MRI data sets were segmented using the FCM algorithm into four different regions representing the brain tissue, skull, air and nasal sinuses. The density $\rho\left(\mathrm{g} / \mathrm{cm}^{3}\right)$ was set to 1.04 for brain tissue, ${ }^{23} 1.6$ for the cranium, ${ }^{24}$ and an average of 0.6 was assigned to nasal sinuses. ${ }^{25}$ This latter value was confirmed by region-of-interest (ROI) analysis of x-ray CT patient data. The mass attenuation coefficients for these tissues were calculated for an energy of $511 \mathrm{keV}$ by means of the XCOM photon cross-section library ${ }^{26}$ using human tissue composition data from ICRU 44 report. ${ }^{23}$ The linear attenuation coefficients are then obtained by multiplying the mass attenuation coefficients by the density of the corresponding tissue. This corresponds to $0.0993,0.143$, and $0.0536 \mathrm{~cm}^{-1}$ for brain tissue, skull and nasal sinuses, respectively. Attenuation in air cavities was neglected. The boundaries are then made more natural by applying a $5 \times 5$ Gaussian filter. Table I summarizes linear attenuation coefficients reported in the literature for energies of interest in nuclear medicine imaging (SPECT and PET) and different attenuating tissues encountered in cerebral scanning. The assumptions made regarding the skull thickness are also shown. It is worth emphasizing that data related to SPECT imaging were limited to references considering the nasal sinuses.

\section{Patient data acquisition}

\section{PET imaging}

The developed attenuation correction algorithm was tested on clinical data sets obtained on the whole body 3D only ECAT ART tomograph (CTI/Siemens, Knoxville, TN, USA). This is a continuously rotating commercial partialring cylindrical positron tomograph upgraded to use collimated single-photon point sources of ${ }^{137} \mathrm{Cs}$ to produce high quality transmission data as consequence of higher count rates resulting from the decreased detector dead time and improved object penetration in comparison to conventional positron-emitting transmission sources. ${ }^{27}$ The reconstructed images consist of 47 slices with $128 \times 128$ resolution (voxel size $=3.4 \times 3.4 \times 3.4 \mathrm{~mm}^{3}$ ).

Clinical brain PET scans of 10 patients referred to the Nuclear Medicine Division of Geneva University Hospital for detection of epileptic foci with seizures using $\left[{ }^{18} \mathrm{~F}\right]$-Fluorodeoxyglucose $(\mathrm{FDG})$ were selected from the database and used for clinical evaluation of the developed attenuation correction method. A preinjection TX measurement (10 min) was performed for attenuation correction purposes as used in clinical routine and served as gold standard to evaluate the developed method. The TX data are normalized to a slab phantom scan and corrected for scatter and cross section variation using a log-linear transformation of the attenuation factors. ${ }^{27}$ The emission study $(25 \mathrm{~min})$ started 30 min after intravenous injection of approximately $222 \mathrm{MBq}$ of $\left[{ }^{18} \mathrm{~F}\right]-\mathrm{FDG}$.

\section{MR imaging}

We defined the anatomy of each patient using highresolution 3D T1-weighted volumetric MR scanning performed with a 1.5-Tesla Eclipse scanner (Philips Medical Systems, Best, The Netherlands). Transaxial and coronal 3D T1-weighted images were acquired with a $1 \mathrm{~mm}$ slice thickness and no slice gap. The parameters were as follows: $\mathrm{TR}=15 \mathrm{~ms}, \mathrm{TE}=4.4 \mathrm{~ms}, \mathrm{TI}=300 \mathrm{~ms}$, flip angle $=25$ degrees, matrix $=256 \times 256 \times 160$. The MR images had a transaxial resolution of $0.97 \mathrm{~mm}$ and an axial resolution of $1.1 \mathrm{~mm}$ for a field-of-view corresponding to $250 \times 250 \mathrm{~mm}$.

\section{Attenuation and scatter corrections and image reconstruction}

Scatter correction was performed using a model-based scatter correction algorithm which combines both the emission scan and attenuation map together with the physics of Compton scattering to estimate the scatter distribution. ${ }^{28} \mathrm{~A}$ new numerical implementation of the single-scatter simulation (SSS) algorithm, which is faster than the previous implementation, currently requiring less than $30 \mathrm{~s}$ execution time per bed position for an adult thorax was used in this work. ${ }^{29}$ This new implementation partially takes into account 


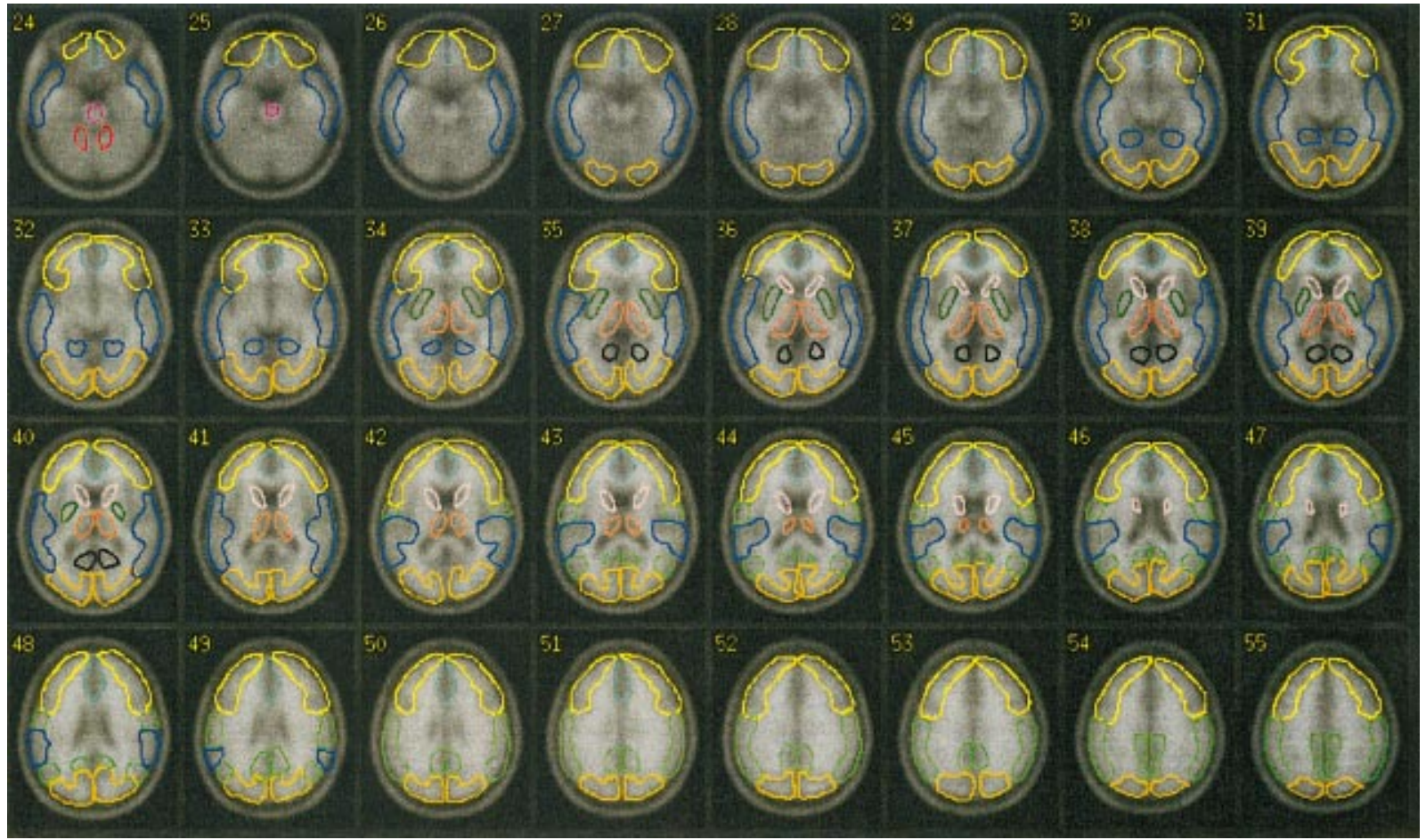

FIG. 3. Transverse slices of the anatomically standardized stereotactic template for automated VOI quantification. The 20 VOIs defined for the quantitative evaluation of the MRI-guided attenuation and scatter corrections are overlaid on the template.

multiple scatter and elegantly solves the normalization problem. In the previous implementation, multiple scatter was compensated by the sinogram scaling. However there may be substantial compensation for multiple scatter even in the absence of scaling when the scatter contribution is computed from an uncorrected estimated image. This hypothesis implies that the total scatter contribution can be estimated by applying the single scatter operator to the uncorrected image, which is the basis of the new algorithm.

Briefly the processing of the data sets involved the following steps: the emission sinogram, TX-based attenuation correction matrix and normalization scan together with the T1-weighted MR brain images were obtained for all data sets. The MR images were thus processed and segmented using the FCM technique as described in Secs. II A and II B. The MRI-guided attenuation correction matrix is then calculated by forward projection at appropriate angles of the resulting attenuation map. The generated ACFs map was then used to correct the emission data. Therefore, the segmented MRI-guided attenuation map served for both scatter and attenuation correction purposes. Both data sets were reconstructed using the reprojection algorithm $(3 \mathrm{DRP})^{30}$ with a maximum acceptance angle corresponding to 17 rings and a span of 7. The default parameters used in clinical routine were applied (Ramp filter, cutoff frequency 0.35 cycles/ pixel).

\section{E. Model validation}

Qualitative and quantitative evaluation of differences between images processed using both TX- and segmented
MRI-guided protocols were performed by visual assessment performed by expert physicians and by estimating parameters of clinical interest including regional cerebral glucose metabolism (rCGM). Transaxial and coronal slices of both data sets were displayed on the computer screen without any identification beside a code not known by the two observers involved in the evaluation. Each observer had to attribute blindly a quality index scaling. Agreement between observers was absolute to identify MRI-guided attenuation correction method as the one providing the best visual image quality. Powerful receiver operating characteristic (ROC)-based analysis of clinical data was not considered in this work and remains to be investigated. The images reconstructed using both processing protocols were realigned to an anatomically standardized stereotactic template using the AIR 3.3 software with a nine-parameter rigid body transformation for automated volume-of-interest (VOI) quantification. Twenty VOIs were defined on different slices of the MRI template by an experienced neuropsychologist and superimposed on each study resulting in a total of 200 VOIs for the 10 patients studied. These are shown overlaid on the MRI template (Fig. 3 ). The co-registered PET images are then used for quantitative analysis using the defined VOIs.

The correlation between mean rCGM estimates obtained when using the two attenuation correction methods was checked on a VOI by VOI basis and using pooled VOI analysis. The means, standard errors and standard deviations of rCGM from PET images reconstructed using both protocols were compared. Statistical analysis was performed VOI by VOI using repeated ANOVA to assess the significance of the 


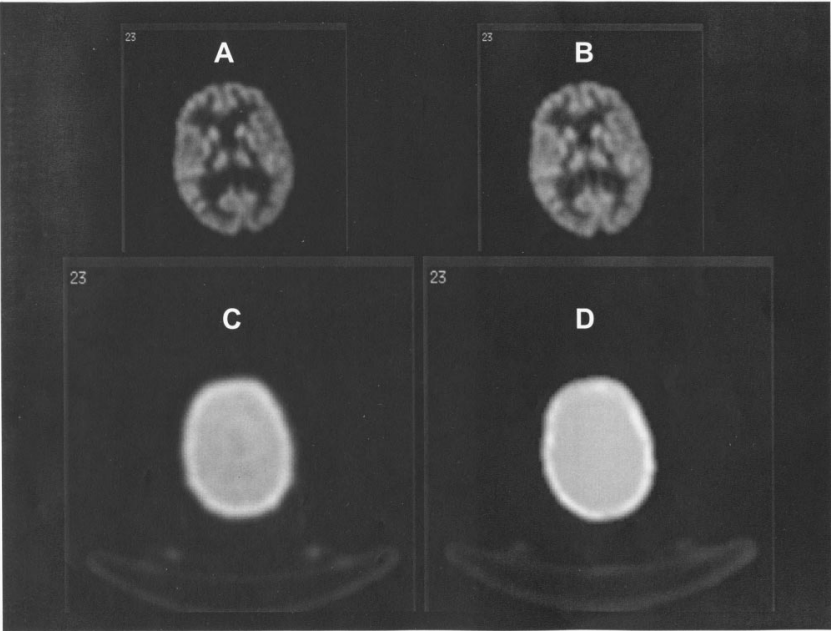

FIG. 4. Comparison of attenuation maps and PET image slices of a patient study reconstructed using the two different processing protocols. (A) PET image reconstructed using transmission-guided attenuation and scatter corrections. (B) PET image reconstructed using segmented MRI-guided attenuation and scatter corrections. (C) Measured transmission-based attenuation map. (D) MRI-guided attenuation map.

differences between mean rCGM estimates in patient studies when using the segmented MRI-guided as compared to the transmission-guided reconstructions (significant $P$ value $<0.05)$. It is worth emphasizing that failure to prove statistically significant differences is not sufficient to confirm that the results are statistically identical.

\section{RESULTS}

\section{A. Qualitative assessment}

Typical patient brain attenuation maps and corresponding PET images acquired with the ECAT ART camera and reconstructed with measured transmission as well as MRI-guided attenuation and scatter corrections are shown in Fig. 4. Both correction methods improve the quality of the images and allow a better definition of brain structures and better overall contrast between gray and white matter compared to the case where no correction is applied; however, the images appear noisier when using transmission-guided attenuation correction. In our nuclear medicine clinic, measured transmissionbased attenuation correction is applied routinely but the physicians preferred the images obtained with the segmented MRI-guided attenuation correction and judged image quality to be much superior. It should be noted that the long acquisition time during TX scanning allowed to drastically improve the signal-to-noise ratio and reduce noise propagation from TX to EM scanning. Horizontal profiles drawn through the thalamus of the slices shown in Fig. 4 are illustrated in Fig. 5. Note the good agreement between measured TXguided and segmented MRI-guided reconstructions causing only small differences between the two distributions. The difference of the profiles is also shown on the same plot. The difference appears more significant on the left side of the profile. This can be explained by the rotational (azimuthal

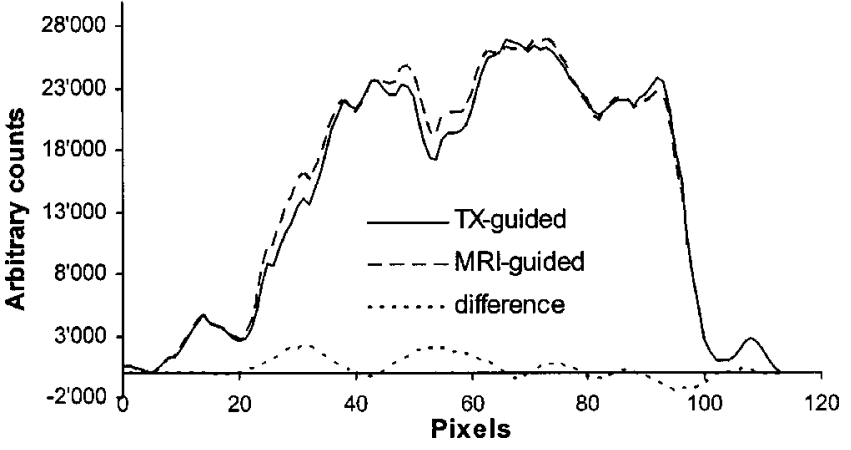

Fig. 5. Comparison of horizontal profiles between images reconstructed using transmission-(solid line) and segmented MRI-guided (shaded line) attenuation and scatter corrections. The difference of the profiles is also shown on the same plot.

angle) misregistration between the emission and transmission images. Note also the better registration between MRIguided attenuation map and emission image.

\section{B. Quantitative analysis}

Figure 6 illustrates the means, standard errors as well as standard deviations resulting from the quantitative analysis for both reconstruction methods in each of the 20 VOIs, averaged across the 10 patients studied. The mean absolute difference for all VOIs across the whole population was $2.4 \%$. Correlation for all VOIs and all patients was found to be high. Figure 7 shows a linear regression plot illustrating correlation between the two attenuation and scatter correction algorithms. The line connecting the data points represents the result of a linear regression analysis. There is a very good correlation $\left(R^{2}=0.91\right)$ between the segmented MRI and measured transmission-guided attenuation and scatter correction techniques and the regression line agreed well with the line of identity (slope $=0.91$ ). The dispersion of data points is insignificant and the general trend as shown by the regression line is that the coefficients of variations are similar. However, segmented MRI-guided attenuation and scatter corrections lead to higher overall estimates than measured attenuation and scatter corrections. This would appear to result from a difference in the measured (TX) and assigned (MRI) $\mu$ values in both methods and thus the attenuation correction factors applied. According to these data, the theoretical attenuation coefficients appear to be higher overall than estimates obtained from measured data. Moreover, our analysis suggests that the intercept in the regression line is significantly different from zero.

The statistical comparison between rCGM estimates when using the two different attenuation and scatter correction techniques is summarized in Table II. The percent differences between the correction techniques are minor but statistically significant for some regions whereas no proof of statistically significant differences could be verified for the other regions. The right and left thalamus as well as right cerebellum and pons regions showed the highest differences and highest probability of a true difference ("the two distributions are not the same"). Figure 8 shows the relative dif- 


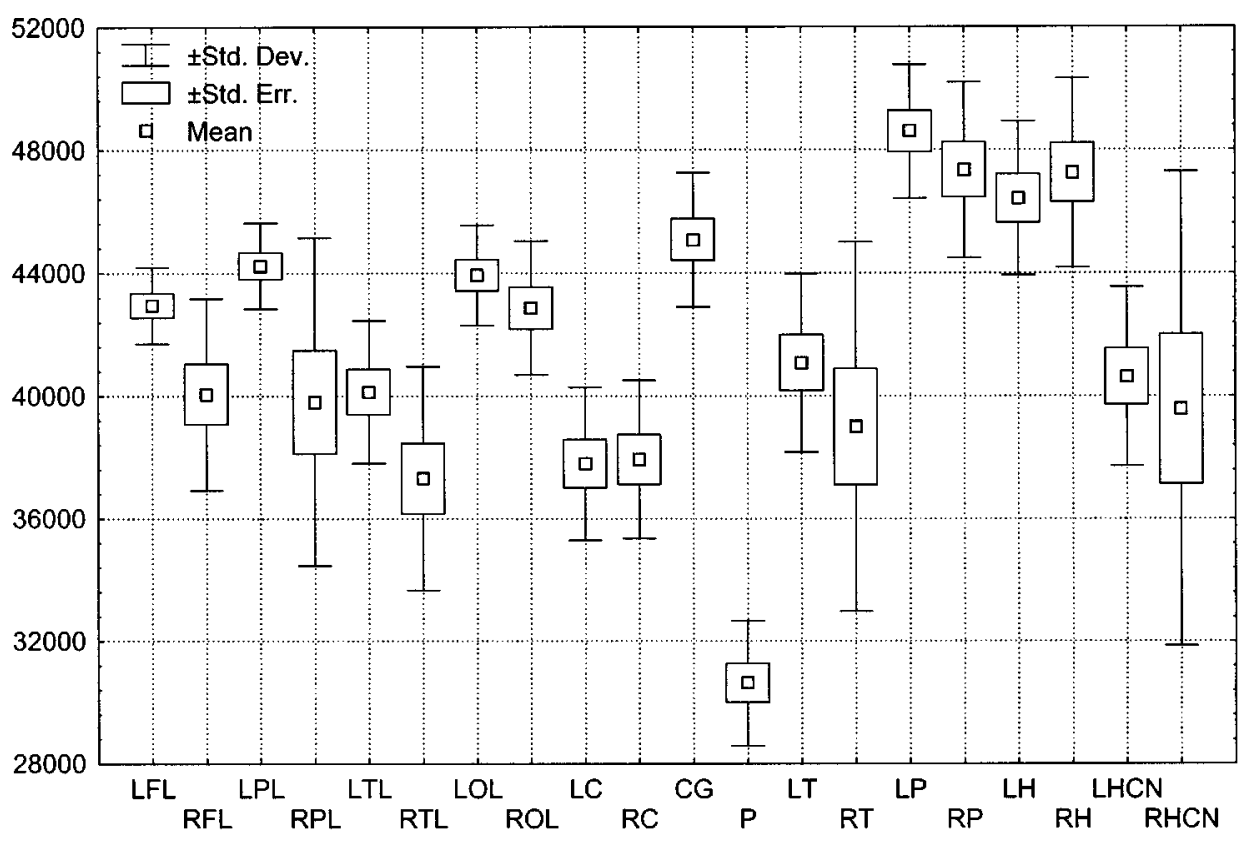

(a)

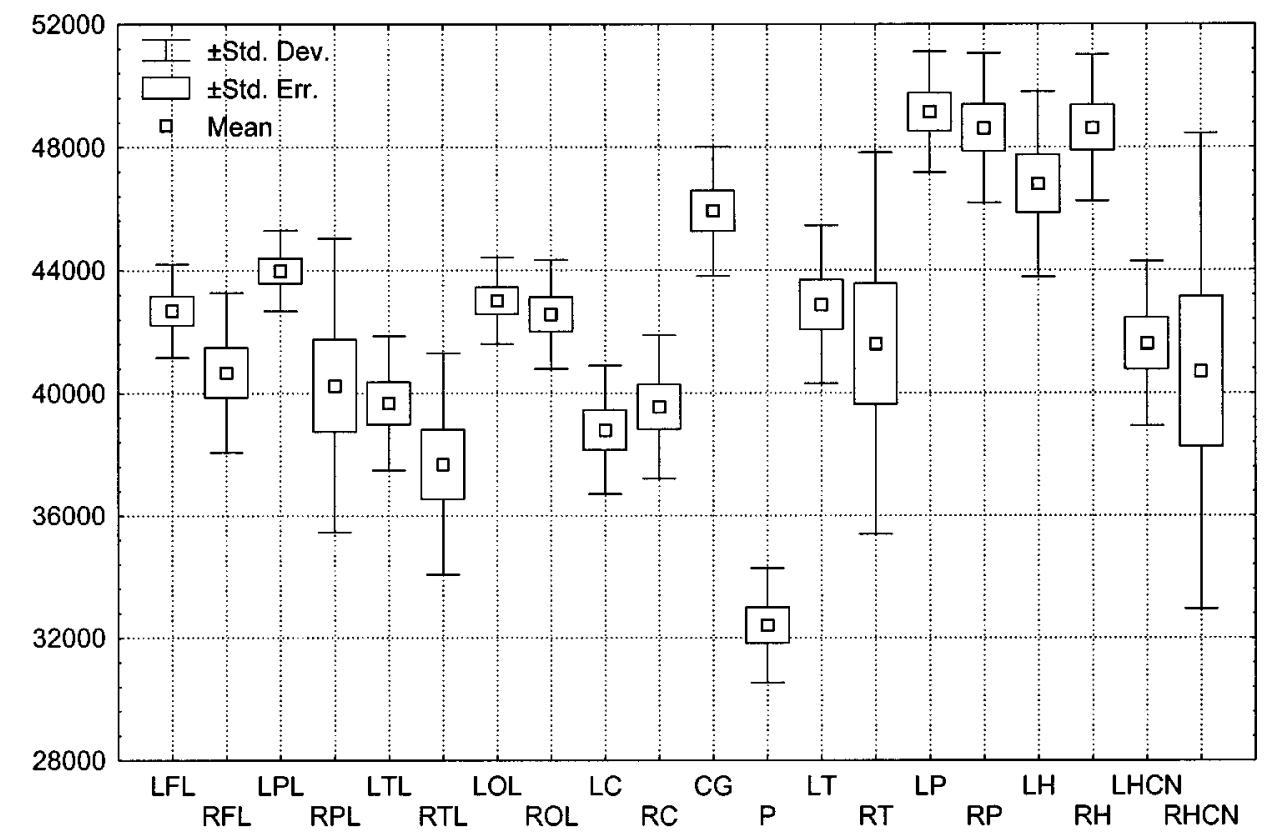

FIG. 6. rCGM estimates are shown for the 20 VOIs. Means, standard errors as well as standard deviations are calculated across the 10 patients studied. Quantification was performed after applying attenuation and scatter corrections guided by measured transmission (a) and segmented MRI (b). On average, reconstructions guided by segmented MRI tend to overestimate rCGM; an overestimation which proved to be statistically significant in nine regions.

(b)

ference between absolute rCGM estimates for clinical brain $3 \mathrm{D}$ reconstructions using attenuation and scatter corrections guided by measured transmission and 3D reconstructions guided by segmented MRI. The relative difference is defined as

Percent difference

$$
=\frac{\mathrm{VOI}(\text { Segmented MRI })-\mathrm{VOI}(\text { Measured TX })}{\mathrm{VOI}(\text { Measured TX })} \times 100 \% .
$$

\section{DISCUSSION}

The quantitative potential of PET imaging relies on accurate procedures for performing attenuation ${ }^{4}$ and scatter ${ }^{31}$ corrections. Various approximate methods have been proposed to solve the problem of photon attenuation in cerebral PET imaging. ${ }^{5-9}$ In our department, when a transmission scan is not available or could not be acquired because of lack of camera time, we use calculated attenuation correction method, which involves user interaction to draw an ellipse approximating the contour of the head on each image of a preliminary two-dimensional reconstruction without attenua- 


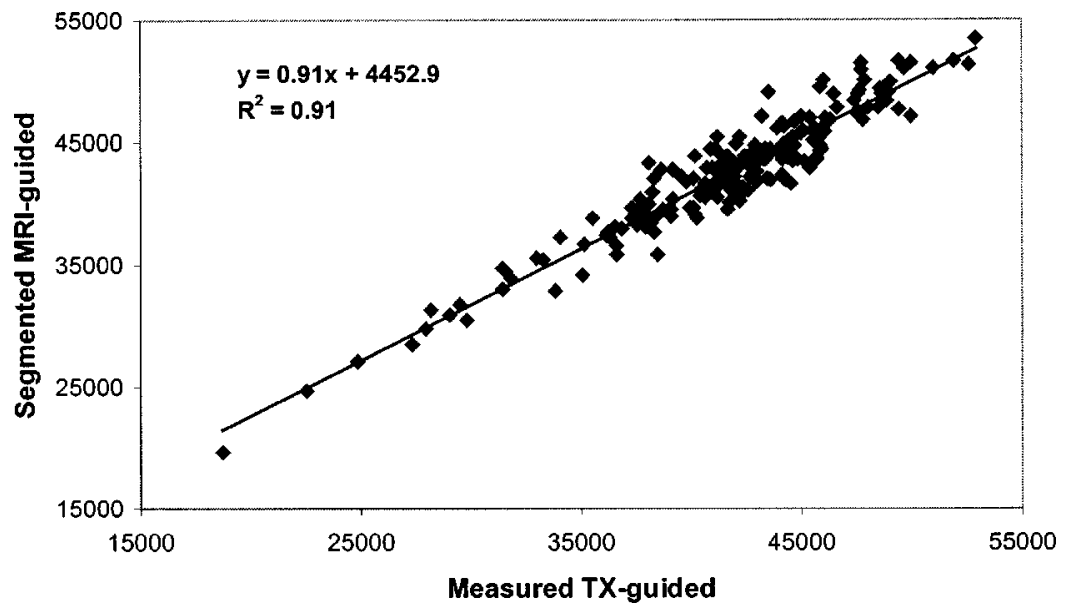

FIG. 7. Correlation plots between clinical 3D brain scans reconstructed using attenuation and scatter corrections guided by measured transmission (abscissa) and 3D reconstructions guided by segmented MRI (ordinate). Two hundred data points representing mean values of the 20 VOIs resulting from the analysis of the 10 patients are shown together with best fit equations and correlation coefficients.

tion correction. Three major drawbacks of this method are: (i) it is time consuming requiring two reconstructions per data set; (ii) it is labor intensive and error prone since the user subjectively defines the ellipse size and location that better fits the head contour; and more importantly (iii) nonuniformities within the head (e.g., skull and nasal sinuses) are not specifically modeled.

The use of transmission-based nonuniform attenuation correction can supply more accurate absolute quantification as compared to calculated attenuation correction and therefore constitutes the gold standard in cerebral 3D PET imaging. However, the necessity of acquiring an additional transmission scan is a restrictive factor for patient throughput. While keeping the total acquisition time constant, simultaneous EM/TX scanning suffers from cross-contamination problems. More importantly, elimination of the TX scan re- sults in reduction in overall radiation absorbed dose, a significant issue when dealing with human volunteers participating in research protocols. A rough estimation of the effective dose equivalent resulting from the additional TX scan led to approximately $63 \mu \mathrm{Sv} .^{32}$ Therefore, a dramatic simplification of PET scanners' design and acquisition protocols could be made if the attenuation map could be obtained without the use of a transmission scan considering the associated design complexity and increased cost in addition to the complicated logistics in clinical routine management. Much worthwhile research and development efforts are devoted to the area of transmissionless attenuation correction. As consequence, a wide array of approaches have been described in the literature; each has its merits and drawbacks. Promising approaches belonging to this class of methods rely on sophisticated algorithms based on statistical modeling for

TABLE II. Summary of statistical assessment using repeated ANOVA analysis for comparing rCGM estimates obtained from clinical brain PET reconstructions guided by measured transmission with reconstructions guided by segmented MRI.

\begin{tabular}{lccc}
\hline \hline \multicolumn{1}{c}{ Volume of interest } & $\begin{array}{c}\text { ANOVA } \\
\text { significance }\end{array}$ & $\begin{array}{c}\text { Paired sample } \\
\text { correlation }\end{array}$ & $\begin{array}{c}\text { Paired sample } \\
\text { significance }\end{array}$ \\
\hline Left frontal lobe (LFL) & 0.502 & 0.644 & 0.045 \\
Right frontal lobe (RFL) & 0.191 & 0.997 & $<0.001$ \\
Left parietal lobe (LPL) & 0.413 & 0.999 & $<0.001$ \\
Right parietal lobe (RPL) & 0.323 & 0.992 & $<0.001$ \\
Left temporal lobe (LTL) & 0.154 & 0.961 & $<0.001$ \\
Right temporal lobe (RTL) & 0.488 & 0.898 & 0.002 \\
Left occipital lobe (LOL) & 0.008 & 0.848 & 0.010 \\
Right occipital lobe (ROL) & 0.517 & 0.765 & 0.001 \\
Left cerebellum (LC) & 0.020 & 0.892 & 0.003 \\
Right cerebellum (RC) & 0.007 & 0.826 & 0.101 \\
Cingulate gyrus (CG) & 0.222 & 0.549 & 0.004 \\
Pons (P) & $0.001^{\mathrm{a}}$ & 0.817 & $<0.001$ \\
Left thalamus (LT) & $0.002^{\mathrm{a}}$ & 0.875 & 0.003 \\
Right thalamus (RT) & $<0.001^{\mathrm{a}}$ & 0.977 & 0.004 \\
Left putamen (LP), & 0.190 & 0.835 & 0.001 \\
Right putamen (RP) & 0.037 & 0.815 & 0.034 \\
Left hippocampus (LH) & 0.454 & 0.869 & 0.001 \\
Right hippocampus (RH) & 0.092 & 0.669 & 0.881 \\
Left head of caudate nucleus (LHCN) & 0.053 & 0.990 & 001 \\
Right head of caudate nucleus (RHCN) & 0.009 & \\
\hline \hline
\end{tabular}

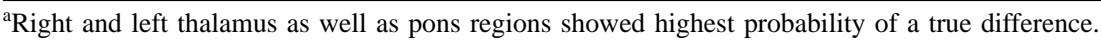




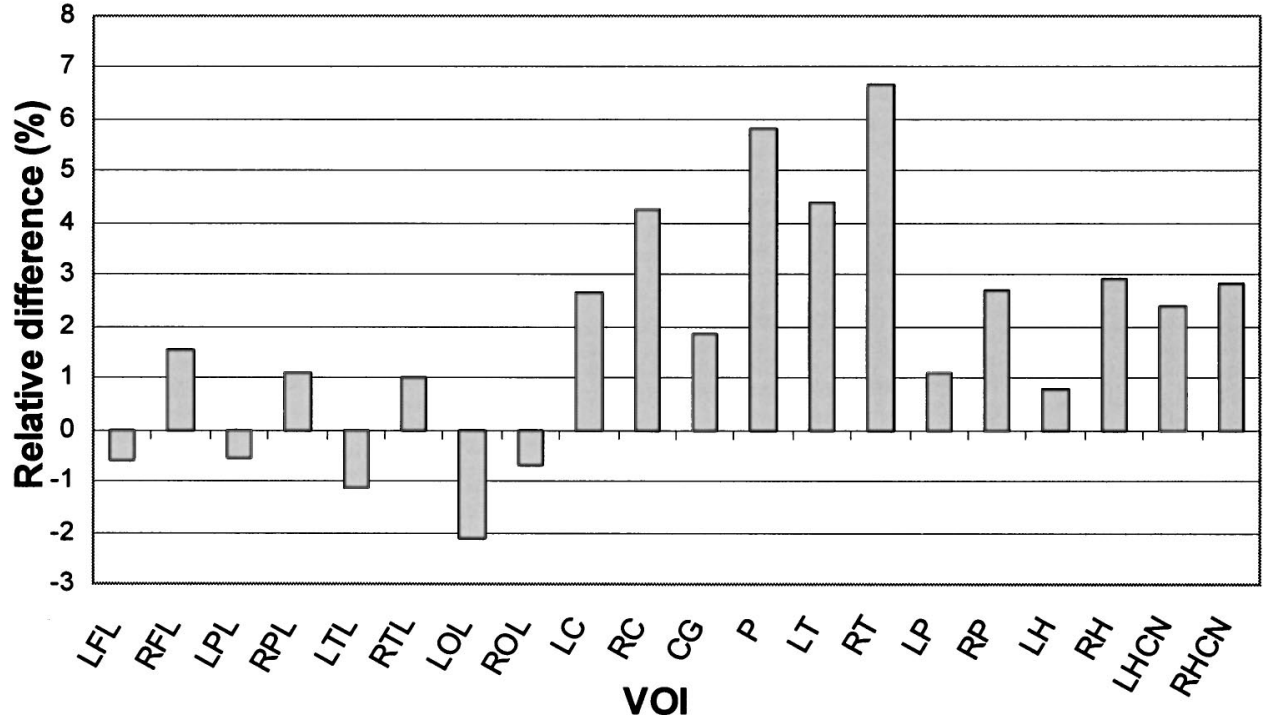

FIG. 8. Relative difference between absolute rCGM estimates for clinical brain 3D reconstructions using attenuation and scatter corrections guided by measured transmission and 3D reconstructions guided by segmented MRI. simultaneous estimation of attenuation and emission distributions or alternatively consistency conditions criteria. In the former case, the problem is formulated as an optimization task where the objective function is a combination of the likelihood and an a priori probability. ${ }^{33}$ The latter uses a Gibbs prior distribution to encourage local smoothness and a multimodal distribution for the attenuation coefficients. In spite of limited success, methods of simultaneous reconstruction often cause artifacts in the form of cross-talk between the activity image and the attenuation map. Quite different results could be obtained if the attenuation map would be reconstructed without finding the activity image.

A more general technique was introduced by Natterer, ${ }^{34}$ who applied continuous consistency conditions for the range of the attenuated Radon transform to derive the attenuation map from emission data. Encouraging results were obtained enabling at least to find the uniform elliptical attenuation distribution, which is most consistent with the measured projections. Bronnikov ${ }^{35}$ strengthened the paradigm of the consistency conditions by setting them in the framework of a discrete representation of the problem. Such an approach ensures a natural regularization of the problem, allowing one to use the well-known method of Tikhonov regularization. Moreover, one of the main advantages of this method over previously used continuous conditions is that it can easily be applied in various scanning configurations, including fully 3D data acquisition protocols. This method has a stable numerical implementation, allowing to avoid the crosstalk between the attenuation map and the source distribution. Nevertheless, the algorithms' evaluation was limited to simulated and/or experimental phantom studies. Consequently, there is still a lack of confidence in their applicability in a clinical setting.

A different approach to the problem was considered in this paper to compute the attenuation map without transmission measurements taking advantage of the availability and ease of access through the hospital picture archiving and communications system of the T1-weighted MR images for patients undergoing brain PET studies. One of the major advantages of our approach compared to the conventional transmission-based method is that in this latter case, there is poor contrast in attenuation coefficients between bone and soft tissue at $511 \mathrm{keV}$ for short transmission scans. Notwithstanding, MRI is good at looking at the nonbony parts or "soft tissues" of the head (brain, spinal cord and nerves), whereas X-ray CT is better at looking at the bones. An equivalent method substituting the patient-specific MR images with a coregistered digitized head atlas derived from high-resolution MRI-based voxel head model ${ }^{11}$ called inferring-attenuation distributions has been proposed for brain SPECT $^{10}$ and extended later for brain PET imaging. ${ }^{36}$ As recognized by the authors, a major difficulty lies in matching patient-specific anatomy with the brain atlas and the lack of precise anatomic details in certain regions. An example of an anomaly associated with the Zubal-based anatomic atlas ${ }^{11}$ is the nasal sinus, which is substantially larger than usual and therefore misrepresentative of the patient population. ${ }^{10,25}$ More elaborate voxel-based phantoms might help overcoming this difficulty though the sinus cavities are the most dissimilar structures in the anatomy of the head among a given population.

The proposed attenuation and scatter correction scheme involves many processing steps and requires acquisition of an MRI scan to define the anatomical size and tissue composition of the different structures contained within the head. These additional steps are costly in expense and time, and may introduce further errors. For instance, co-registration between PET and MRI images has an accuracy limit of less than $2 \mathrm{~mm},{ }^{18}$ which in our opinion is better that uncertainties associated to misregistration between emission and preinjection TX images (see angular misregistration between transmission and emission images in Fig. 4). A major difficulty in the segmentation of MR images is the intensity inhomogeneity artifact, which causes a shading effect to appear over the image. This artifact can significantly degrade the performance of methods which assume that the intensity value of a 
tissue class is constant over the image. Because FCM does not place any contextual constraints on the membership functions it can be sensitive to excessive noise and other artifacts, such as intensity inhomogeneities. In agreement with current literature, we found that for spin-echo acquisitions, the inhomogeneity was nearly identical for different pulse echo times (TE).

It is recognized that our method was validated using limited clinical data. It would be interesting to evaluate the developed attenuation correction method using a fully tissueequivalent anthropomorphic head phantom like the one manufactured by RSD (Radiology Support Devices, Long Beach, CA). A known limitation of the method is that the thermoplastic mask used for reproducible patient repositioning between preinjection TX and EM scanning and head holder were not explicitly modeled. No attempt was made to correct intensity inhomogeneities typically caused by nonuniformities in the RF field during MRI acquisition, in addition to many other effects. As mentioned previously, the difficulties associated with automatic segmentation of the skull on the T1-weighted images using the FCM algorithm led to some manual intervention of the operator. Multispectral MR data acquisition with varying contrast characteristics including spin-echo T2-weighted and proton spin density (PD)weighted provides additional information for distinguishing between different tissues and could be an appealing approach to solve this problem.

While accurate attenuation correction has been the primary objective for determining the attenuation map in PET, there are many other potential applications especially when it is derived from high-resolution anatomical images. Typical applications include partial-volume correction and image fusion of anatomical and functional data to facilitate VOI delineation for quantitative analysis as well as anatomically guided image reconstruction. ${ }^{4}$ More elaborated algorithms integrating all physical degradation phenomena (attenuation, scatter, partial-volume effect, etc.) into a unified statistical reconstruction algorithm combining anatomical MRI and functional PET data remains to be explored.

\section{CONCLUSION}

The segmented MRI-based approach is suitable for clinical routine application in 3D brain PET imaging where the MR data are usually available. The results indicated a clear improvement in image quality as a consequence of the reduction of noise propagation from transmission into emission data. The elimination of the transmission scan allows to reduce misalignment errors due to patient motion when using a preinjection TX scanning protocol, reduce patient absorbed dose and shorten the total acquisition time resulting in more comfort to patients (especially those with neurodegenerative disease) and a substantial increase in patient throughput. Considering the difficulties associated with transmissionbased attenuation correction and the limitations of current calculated attenuation correction, MRI-based attenuation correction in 3D brain PET would likely be the method of choice for the foreseeable future as a second best approach in a busy nuclear medicine center and could be applied to other functional imaging modalities such as SPECT.

\section{ACKNOWLEDGMENTS}

The authors would like to thank Manuel Diaz-Gomez for performing the analysis of the data sets. This work was supported by the Swiss National Science Foundation under Grant No. SNSF 3152-062008.

${ }^{a)}$ Electronic mail: habib.zaidi@hcuge.ch

${ }^{1}$ M. Watanabe et al., "A new high-resolution PET scanner dedicated to brain research," IEEE Trans. Nucl. Sci. 49, 634-639 (2002).

${ }^{2}$ K. Wienhard et al., "The ECAT HRRT: performance and first clinical application of the new high resolution research tomograph," IEEE Trans. Nucl. Sci. 49, 104-110 (2002)

${ }^{3}$ H. Zaidi and V. Sossi, "Correction for image degrading factors is essential for accurate quantification of brain function using PET," Med. Phys. (in press).

${ }^{4}$ H. Zaidi and B. H. Hasegawa, "Determination of the attenuation map in emission tomography," J. Nucl. Med. 44, 291-315 (2003).

${ }^{5} \mathrm{M}$. Bergstrom et al., "Determination of object contour from projections for attenuation correction in cranial positron emission tomography," $\mathrm{J}$. Comput. Assist. Tomogr. 6, 365-372 (1982).

${ }^{6} \mathrm{~T}$. Tomitani, "An edge detection algorithm for attenuation correction in emission CT,” IEEE Trans. Nucl. Sci. 34, 309-312 (1987).

${ }^{7} \mathrm{C}$. Michel et al., "Online brain attenuation correction in PET: towards a fully automated data handling in a clinical environment," Eur. J. Nucl. Med. 15, $712-718$ (1989).

${ }^{8}$ S. Siegel and M. Dahlbom, "Implementation and evaluation of a calculated attenuation correction for PET," IEEE Trans. Nucl. Sci. 39, 11171121 (1992).

${ }^{9}$ B. T. Weinzapfel and G. D. Hutchins, "Automated PET attenuation correction model for functional brain imaging," J. Nucl. Med. 42, 483-491 (2001).

${ }^{10}$ R. Z. Stodilka et al., "Scatter and attenuation correction for brain SPECT using attenuation distributions inferred from a head atlas," J. Nucl. Med. 41, 1569-1578 (2000).

${ }^{11}$ I. G. Zubal et al., "Computerized 3-dimensional segmented human anatomy," Med. Phys. 21, 299-302 (1994).

${ }^{12} \mathrm{H}$. Watabe et al., "Acquisition of attenuation map for brain PET study using optical tracking system," Proceedings of the IEEE Nuclear Science Symposium and Medical Imaging Conference, San Diego, CA, 2001, Vol. 3, pp. 1458-1461.

${ }^{13}$ P. E. Kinahan et al., "Attenuation correction for a combined 3D PET/CT scanner," Med. Phys. 25, 2046-2053 (1998).

${ }^{14}$ Y. Shao et al., "Simultaneous PET and MR imaging," Phys. Med. Biol. 10, 1965-1970 (1997).

${ }^{15}$ R. B. Slates et al., "A study of artefacts in simultaneous PET and MR imaging using a prototype MR compatible PET scanner," Phys. Med. Biol. 44, 2015-2027 (1999).

${ }^{16} \mathrm{D}$. W. Townsend and S. R. Cherry, "Combining anatomy and function: the path to true image fusion," Eur. Radiol. 11, 1968-1974 (2001).

${ }^{17}$ H. Zaidi et al., "MRI-guided attenuation correction in 3D brain PET," 8th International Conference on functional mapping of the human brain., June 2-6, Sendai, Japan, Available on CD-Rom in NeuroImage, Vol. 16, No. 2, Abstract 504, 2002.

${ }^{18}$ R. P. Woods et al., "Automated image registration: II. Intersubject validation of linear and nonlinear models," J. Comput. Assist. Tomogr. 22, 153-165 (1998).

${ }^{19}$ J. C. Bezdek et al., "Convergence theory for fuzzy c-means: counterexamples and repairs," IEEE Trans. Syst. Man Cybern. 17, 873-877 (1987).

${ }^{20}$ J. C. Bezdek, L. O. Hall, and L. P. Clarke, "Review of MR image segmentation techniques using pattern recognition," Med. Phys. 20, 10331048 (1993).

${ }^{21}$ H. Zaidi et al., "Fuzzy clustering-based segmented attenuation correction in whole-body PET imaging," Phys. Med. Biol. 47, 1143-1160 (2002).

${ }^{22}$ A. O. Boudraa et al., "Automated segmentation of multiple sclerosis lesions in multispectral MR imaging using fuzzy clustering," Comput. Biol. Med. 30, 23-40 (2000). 
${ }^{23}$ ICRU, International Commission on Radiological Units and Measurements (ICRU), Report No. 44, 1989.

${ }^{24}$ D. R. White, H. Q. Woodard, and S. M. Hammond, "Average soft-tissue and bone models for use in radiation dosimetry,” Br. J. Radiol. 60, 907913 (1987).

${ }^{25}$ A. Arlig et al., "Attenuation correction in quantitative SPECT of cerebral blood flow: a Monte Carlo study," Phys. Med. Biol. 45, 3847-3859 (2000).

${ }^{26}$ M. J. Berger and J. H. Hubbell, National Bureau of Standards (US); Report No. NBSIR 87-3597, 1987.

${ }^{27}$ C. C. Watson et al., "Design and performance of a single photon transmission measurement for the ECAT ART," Proceedings of the IEEE Nuclear Science Symposium and Medical Imaging Conference, 1997, Vol. 2, pp. 1366-1370.

${ }^{28}$ C. C. Watson et al., "Evaluation of simulation-based scatter correction for 3-D PET cardiac imaging," IEEE Trans. Nucl. Sci. 44, 90-97 (1997).

${ }^{29}$ C. C. Watson, "New, faster, image-based scatter correction for 3D PET," IEEE Trans. Nucl. Sci. 47, 1587-1594 (2000).
${ }^{30}$ P. E. Kinahan and J. G. Rogers, “Analytic 3D image reconstruction using all detected events," IEEE Trans. Nucl. Sci. 36, 964-968 (1989).

${ }^{31}$ H. Zaidi, "Scatter modelling and correction strategies in fully 3D PET," Nucl. Med. Commun. 22, 1181-1184 (2001).

${ }^{32}$ A. Schaefer et al., "Radiation exposure to patients caused by singlephoton transmission measurement in PET [German]," Nuklearmedizin 39, 204-208 (2000).

${ }^{33} \mathrm{~J}$. Nuyts et al., "Simultaneous maximum a posteriori reconstruction of attenuation and activity distributions from emission sinograms," IEEE Trans. Med. Imaging 18, 393-403 (1999).

${ }^{34}$ F. Natterer, "Determination of tissue attenuation in emission tomography of optically dense media," Inverse Probl. 9, 731-736 (1993).

${ }^{35}$ A. V. Bronnikov, "Reconstruction of attenuation map using discrete consistency conditions," IEEE Trans. Med. Imaging 19, 451-462 (2000).

${ }^{36}$ H. Zaidi, M-L. Montandon, and D. O. Slosman, "Comparison of six attenuation correction schemes in cerebral 3D positron emission tomography," Conference Proceedings of the 2nd European Medical \& Biological Engineering Conference (EMBEC'02), Vienna (Austria), December 04-08, 2002, Vol. 2, pp. 868. 